International Journal of Pure and Applied Mathematics

Volume 115 No. 3 2017, 607-619

ISSN: 1311-8080 (printed version); ISSN: 1314-3395 (on-line version)

url: http://www.ijpam.eu

doi: 10.12732/ijpam.v115i3.14

ijpam.eu

\title{
A MATHEMATICAL LOGIC VALIDATION PROCEDURE FOR PETRI NETS MODELS
}

\author{
Zvi Retchkiman Königsberg \\ Instituto Politécnico Nacional, CIC \\ Mineria 17-2, Col. Escandon, Mexico D.F 11800, MEXICO
}

\begin{abstract}
A discrete event system, is a dynamical system whose state evolves in time by the occurrence of events at possibly irregular time intervals.. Place-transitions Petri nets, commonly called Petri nets, are a graphical and mathematical modeling tool applicable to discrete event systems in order to represent its states evolution.

This paper proposes a formal modeling and validation mathematical analysis methodology, which consists in representing the Petri net model of a discrete event system, by means of a formula in the propositional calculus logic. Then, using the concept of logic implication, and transforming this logical implication relation into a set of clauses, qualitative methods for validation are addressed.
\end{abstract}

AMS Subject Classification: 03B70, 93A30, 93D99, 93C10

Key Words: Petri net models, discrete event systems, propositional logic, validation, refutation methods

\section{Introduction}

A discrete event system, is a dynamical system whose state evolves in time by the occurrence of events at possibly irregular time intervals. Some examples

Received: $\quad$ May 29, 2017

Revised: July 19, 2017

Published: July 27, 2017

(c) 2017 Academic Publications, Ltd. url: www.acadpubl.eu 
include: Manufacturing systems, Computer networks, Queuing systems, Communication systems, Business processes. Place-transitions Petri nets, commonly called Petri nets, are a graphical and mathematical modeling tool applicable to discrete event systems in order to represent its states evolution. Petri nets are known to be useful for analysing the systems properties in addition of being a paradigm for describing and studying information processing systems. Most often such Petri net models are constructed and implemented without any formal validation analysis or by studying its state space. This paper proposes a formal modeling and validation mathematical analysis methodology, which consists in representing the Petri net model of a discrete event system, by means of a formula in the propositional calculus logic. Then, using the concept of logic implication, and transforming this logical implication relation into a set of clauses, qualitative methods for validation, are addressed. The method of Putnam-Davis based for testing the unsatisfiability of a set of clauses as well as the resolution principle due to Robinson, are invoked. The paper is organized as follows. In Section 2, Petri nets are defined and a propositional calculus background summary which supports the procedure presented in the paper is given. In Section 3, the Putnam-Davis rules and the resolution principle for unsatisfiability, are recalled. In Section 3, we illustrate how the methodology is applied. Finally, the paper ends with some conclusions.

\section{Petri Nets and Propositional Calculus}

Notation. $N=\{0,1,2, \ldots\}, N_{n_{0}}^{+}=\left\{n_{0}, n_{0}+1, \ldots, n_{0}+k, \ldots\right\}, n_{0} \geq 0$.

A Petri net $(P N)$ is a 5-tuple, $P N=\left\{P, T, F, W, M_{0}\right\}$ where: $P=\left\{p_{1}, p_{2}, \ldots\right.$, $\left.p_{m}\right\}$ is a finite set of places, $T=\left\{t_{1}, t_{2}, \ldots, t_{n}\right\}$ is a finite set of transitions (represented respectively by circles and bars), $F \subset(P \times T) \cup(T \times P)$ is a set of arcs, $W: F \rightarrow N_{1}^{+}$is a weight function, $M_{0}: P \rightarrow N$ is the initial marking, $P \cap T=\varnothing$ and $P \cup T \neq \varnothing$. Notice that if $W(p, t)=\alpha$ or $W(t, p)=\beta$, then, this is often represented graphically by $\alpha$ or $\beta$ arcs from $p$ to $t$ or $t$ to $p$ (through arrowheads) each with no numeric label. Let $M\left(p_{i}\right)$ denote the marking (i.e., the number of tokens) at place $p_{i} \in P$ and let $M=\left[M\left(p_{1}\right), \ldots, M\left(p_{m}\right)\right]^{T}$ denote the marking (state) of $P N$. A transition $t_{j} \in T$ is said to be enabled if $M\left(p_{i}\right) \geq W\left(p_{i}, t_{j}\right)$ for all $p_{i} \in P$ such that $\left(p_{i}, t_{j}\right) \in F$. If a transition is enabled then, it can fire. If an enabled transition $t_{j} \in T$ fires then, the next marking for $p_{i} \in P$ is given by

$$
M^{\prime}\left(p_{i}\right)=M\left(p_{i}\right)+W\left(t_{j}, p_{i}\right)-W\left(p_{i}, t_{j}\right)
$$




\subsection{Propositional Calculus}

This sub-section presents a summary of the propositional logic theory. The reader is referred to [2] and [3] for details.

Definition 1. (The alphabet for propositional formula) This alphabet consists of:

1. A countable set $P S$ of proposition symbols called atoms: $P_{0}, P_{1}, P_{2}, \ldots$

2. The logical connectives: $\wedge$ (and), $\vee$ (or), $\rightarrow$ (implication), $\sim$ (not), and $\leftrightarrow$ (equivalence).

3. Auxiliary symbols: ( (left parenthesis), ) (right parenthesis).

Definition 2. The set of proposition formula is the smallest set of strings over the alphabet of definition 1, such that:

1. Every proposition symbol $P_{i}$ is a proposition formula

2. Whenever $A$ is a proposition formula, $\sim A$ is also

3. Whenever $A, B$ are proposition formula, $(A \wedge B),(A \vee B),(A \rightarrow B)$ and $(A \leftrightarrow B)$ are also

4. A string is a proposition formula only if it is formed by applying the rules (1), (2), (3).

Definition 3. By an assignment on a given set of atoms we mean a function $v$ which maps each atom into the set FALSE,TRUE $=\{0,1\}$, where we are identifying FALSE with 0 and TRUE with 1 . Thus for each atom $P_{i}$ we will have $v\left(P_{i}\right)=0$ or $v\left(P_{i}\right)=1$.

Definition 4. Given an assignment $v$ on a set of atoms, we define recursively a value $\gamma^{v} \in\{0,1\}$ for each formula $A$ :

1. if $A$ is an atom, then $A^{v}=v(A)$

2. If $\gamma=\sim A$ then $\gamma^{v}= \begin{cases}1 & \text { if } A^{v}=0 \\ 0 & \text { if } \quad A^{v}=1\end{cases}$

3. $(A \vee B)^{v}=\left\{\begin{array}{cc}0 & \text { if } \\ 1 & \text { otherwise }\end{array} A^{v}=B^{v}=0\right.$

4. $(A \wedge B)^{v}=\left\{\begin{array}{cc}1 & \text { if } \\ 1 & \text { otherwise }\end{array} A^{v}=B^{v}=1\right.$ 
5. $(A \rightarrow B)^{v}=\left\{\begin{array}{cc}0 & \text { if } \\ 1 & \text { otherwise }\end{array} A^{v}=1, B^{v}=0\right.$

6. $(A \leftrightarrow B)^{v}=\left\{\begin{array}{cc}1 & \text { if } \\ 0 & \text { otherwise }\end{array} A^{v}=B^{v}\right.$

Definition 5. A formula $A$ is satisfiable if and only if there is an assignment $v$ such that $v(A)=1$ otherwise is said to be unsatisfiable.

Definition 6. A formula $A$ is called a tautology if and only if every assignment of $A$ satisfies it.

Definition 7. A formula $A$ is a logical implication of formulas $F_{1}, F_{2}, \ldots, F_{n}$ if and only if for every assignment $v$, if $F_{1}, F_{2}, \ldots, F_{n}$ is true in $v, A$ is also true in $v$.

The following characterization of logical implication plays a very important role as will be shown in the rest of the paper.

Theorem 8. Given formulas $F_{1}, F_{2}, \ldots, F_{n}$ and a formula $A, A$ is a logical implication of $F_{1}, F_{2}, \ldots, F_{n}$ if and only if the formula $\left(\left(F_{1} \wedge F_{2} \wedge \ldots, \wedge F_{n}\right) \rightarrow A\right)$ is a tautology if and only if the formula $\left(F_{1} \wedge F_{2} \wedge \ldots \wedge F_{n} \wedge \sim(A)\right)$ is unsatisfiable.

Next, Given a formula $A$, the following procedure transforms $A$ into its conjunctive normal form $(\mathrm{CNF})$.

(1) Eliminate $\rightarrow$ and $\leftrightarrow$, (2) Move $\sim$ inward, (3) Rename variables and (4) Pull quantifiers (details are provided in [2]).

Theorem 9. There is an algorithm which transforms any given $A$ formula into a formula $B$ in $C N F$ such that $A=B$.

Definition 10. A clause is a finite disjunction of zero or more literals (atoms or negation of atoms).

We shall regard a set of literals as synonymous with a clause. A clause consisting of $r$ literals is called an $r$-literal clause. A one-literal clause is called a unit clause. When a clause contains no literal, we call it the empty clause, denoted by $\square$. Since the empty clause has no literal that can be satisfied by an assignment, the empty clause is always false. The importance of transforming a formula $A$ into its CNF results evident, thanks to the next result.

Remark 11. Notice that given a formula $A$ in $C N F$, the set of clauses $S$ of $A$ is the representation in terms of sets of its CNF. A set $S$ of clauses is unsatisfiable if and only if it is false under all assignments which implies, thanks to the next result, that $A$ the formula that it represents is unsatisfiable. 
Theorem 12. Let $S$ be a set of clauses that represents a formula $A$. Then a formula $A$ in its $C N F$ form is a tautology if and only if $S$ is a tautology.

We have seen that the problem of logical implication is reducible to the problem of satisfiability, which in turn is reducible to the problem of satisfiability of its CNF, or equivalently to the satisfiability of $S$. This can be used together with algorithms for unsatisfiability, Davis Putnam rules and the resolution principle, (discussed in the next section), to develop procedures for this purpose.

\section{Unsatisfiability Methods}

\subsection{Davis Putnam Rules [2]}

Davis and Putnam introduced a method for testing the unsatisfiability of a set of clauses. Their method consists of the following rules: (1) Delete all clauses from $S$ that are tautologies. The remaining set $S^{\prime}$ is unsatisfiable if and only if $S$ is, (2) If there is a unit clause $L$ in $S$, obtain $S^{\prime}$ from $S$ by deleting those clauses in $S$ containing $L$. If $S^{\prime}$ is empty then, $S$ is satisfiable, otherwise obtain a set $S^{\prime \prime}$ by deleting $\sim(L)$ from $S^{\prime}$. $S^{\prime \prime}$ is unsatisfiable if and only if $S$ is, (3) A literal $L$ in a clause of $S$ is said to be pure in $S$ if and only if the literal $\sim(L)$ does not appear in any clause in $S$. If a literal $L$ is pure in $S$, delete all the clauses containing $L$. The remaining set $S^{\prime}$ is unsatisfiable if and only if $S$ is, (4) If the set $S$ can be written as: $\left(A_{1} \vee L\right) \wedge\left(A_{2} \vee L\right) \ldots\left(A_{m} \vee L\right) \wedge\left(B_{1} \vee \sim\right.$ $L) \wedge\left(B_{2} \vee \sim L\right) \ldots\left(B_{m} \vee \sim L\right) \wedge R$ where $A_{i}, B_{i}$ and $R$ are free of $L$ and $\sim L$ then, obtain the sets $S_{1}=A_{1} \wedge A_{2} \ldots A_{m} \wedge R$ and $S_{2}=B_{1} \wedge B_{2} \ldots B_{m} \wedge R$. $S$ is unsatisfiable if and only if both, $S_{1} \cup S_{2}$ are.

\subsection{The Resolution Principle [1]}

We shall next introduce the resolution principle due to Robinson, which is also called the Davis and Putnam procedure in the literature [2]. It can be applied directly to any set $S$ of clauses to test the unsatisfiability of $S$. Resolution is a sound and complete algorithm i.e., a formula in clausal form is unsatisfiable if and only if the algorithm reports that it is unsatisfiable. Therefore it provides a consistent methodology free of contradictions.

Definition 13. Let $C_{1}$ and $C_{2}$ be two clauses (called parent clauses) with no variables in common. Let $L_{1}$ and $L_{2}$ be two literals in $C_{1}$ and $C_{2}$, respectively and consider $L_{1}$ and $\sim\left(L_{2}\right)$, then the clause $\left(C_{1}-L_{1}\right) \cup\left(C_{2}-L_{2}\right)$ 
is called a binary resolvent of $C_{1}$ and $C_{2}$. The literals $L_{1}$ and $L_{2}$ are called the literals resolved upon.

Definition 14. A resolvent of (parent) clauses $C_{1}$ and $C_{2}$ is one of the following binary resolvents: (1) a binary resolvent of $C_{1}$ and $C_{2}$, (2) a binary resolvent of $C_{1}$ and a factor of $C_{2}$, (3) a binary resolvent of a factor of $C_{1}$ and $C_{2}$, (4) a binary resolvent of a factor of $C_{1}$ and a factor of $C_{2}$.

Definition 15. Given a set $S$ of clauses, a deduction of $C$ from $S$ is a finite sequence of clauses $C_{1}, C_{2}, \ldots, C_{n}$ such that each $C_{i}$, either is a clause in $S$ or a resolvent of clauses preceding $C_{i}$, and $C_{k}=C$. A deduction of $\square$ from $S$ is called a refutation, or a proof of $S$.

The main result of this sub-section, the soundness and completeness theorem for the resolution procedure, is next presented.

Theorem 16. A set $S$ of clauses is unsatisfiable if and only if there is a deduction of the empty clause $\square$ from $S$.

\section{Validation of Petri Net Models}

In this section two examples are provided, the machine shop and a more computational challenging example given by the multirobotic system.

\subsection{The Machine Shop System Modeling Problem}

As an example, consider a simple machine shop whose Petri net model is depicted in fig.1.

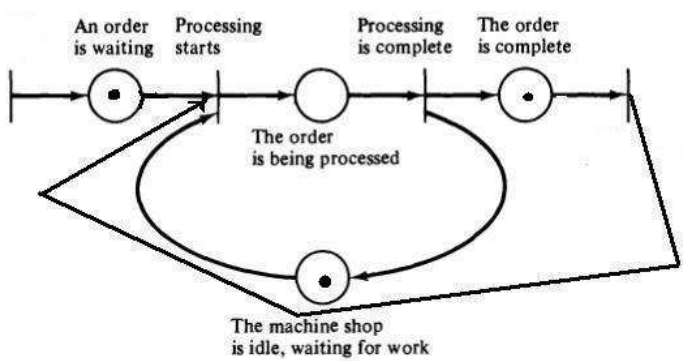

Figure 1. Machine Shop System

The machine shop waits until an order appears and then machines the ordered part and sends it out for delivery. The machine shop behavior as described 
by the Petri net model is as follows: (1) States: $O W B P$ : An order is waiting to being processed, $O B P$ : An order is being processed, $M I$ : The machine is idle, $O C$ : the order is complete; (2) Rules of Inference: (a) if $(O W B P$ ) and $(M I)$ and $(O C)$ then $\operatorname{not}(O B P),(\mathrm{b})$ if $(O B P)$ then $\operatorname{not}(O C)$ and $\operatorname{not}(M I),(\mathrm{c})$ if $(O W B P)$ and $\operatorname{not}(M I)$ then $(O B P),(\mathrm{d})$ if $\operatorname{not}(O B P)$ then $(M I)$ and $(O C)$ and $(O W B P)$.

Remark 17. The main idea consists of: the Petri net model description is expressed by a propositional formula, some validation question is expressed as an additional formula. The question is assumed to be a logical implication of the formula (see theorem 13). Then, transforming this logical implication relation into a set of clauses by using the techniques given in Section 2, its validity can be checked.

The formula that models the machine shop turns out to be:

$$
\begin{array}{r}
{[(O W B P) \wedge(M I) \wedge(O C) \rightarrow \sim(O B P)] \wedge[(O B P) \rightarrow \sim(O C) \wedge \sim(M I)]} \\
\wedge[(O W B P) \wedge \sim(M I) \rightarrow(O B P)] \wedge[\sim(O B P) \\
\rightarrow(M I) \wedge(O C) \wedge(O W B P)] .
\end{array}
$$

We are interested in the following validations:

(S1) Claim: $O B P \rightarrow \sim(M I)$.

The set of clauses is given by:

$$
\begin{array}{r}
S=\{(\sim(O W B P) \vee \sim(M I) \vee \sim(O C) \vee \sim(O B P)),(\sim(O B P) \vee \\
\sim(O C)),(\sim(O B P) \vee \sim(M I)),(\sim(O W B P) \vee(M I) \vee(O B P)),((O B P) \\
\vee(O C)),((O B P) \vee(M I)),((O B P) \vee(O W B P)),(O B P),(M I)\}
\end{array}
$$

Then a resolution refutation proof is as follows:

(a) $(O B P)(\sim(O B P) \vee \sim(M I)) \rightarrow \sim(M I),(\sim M I)(M I) \rightarrow \square$.

(S2) Claim: $\sim(M I) \rightarrow O B P$.

The set of clauses is given by:

$$
\begin{aligned}
S= & \{(\sim(O W B P) \vee \sim(M I) \vee \sim(O C) \vee \sim(O B P)), \\
& (\sim(O B P) \vee \sim(O C)),(\sim(O B P) \vee \sim(M I)),(\sim(O W B P) \vee(M I) \\
& \vee(O B P)),((O B P) \vee(O C)),((O B P) \vee(M I)),((O B P) \vee(O W B P)), \\
& \quad(\sim O B P),(\sim(M I))\} .
\end{aligned}
$$

Then a resolution refutation proof is as follows: 
(a) $((\sim(M I))((O B P) \vee(M I)) \rightarrow O B P,(O B P)(\sim O B P) \rightarrow \square$.

(S3) Claim: $O C \rightarrow M I$.

The set of clauses is given by:

$$
\begin{aligned}
S=\{(\sim(O W B P) \vee \sim(M I) \vee \sim(O C) \vee \sim(O B P)),(\sim(O B P) \\
\vee \sim(O C)),(\sim(O B P) \vee \sim(M I)),(\sim(O W B P) \vee(M I) \\
\vee(O B P)),((O B P) \vee(O C)),((O B P) \vee(M I)), \\
((O B P) \vee(O W B P)),(O C),(\sim(M I))\} .
\end{aligned}
$$

Then a resolution refutation proof is as follows:

(a) $(O C)(\sim(O B P) \vee \sim(O C)) \rightarrow \sim(O B P)$.

(b) $(\sim(M I))((O B P) \vee(M I)) \rightarrow O B P$.

Then, from (a) and (b) we conclude $\square$.

(S4) Claim: $\sim(O C) \rightarrow(O B P \wedge \sim(M I))$

The set of clauses is given by:

$$
\begin{array}{r}
S=\{(\sim(O W B P) \vee \sim(M I) \vee \sim(O C) \vee \sim(O B P)),(\sim(O B P) \\
\vee \sim(O C)),(\sim(O B P) \vee \sim(M I)),(\sim(O W B P) \vee(M I) \vee(O B P)), \\
((O B P) \vee(O C)),((O B P) \vee(M I)),((O B P) \vee(O W B P)),(\sim O C), \\
(M I \vee \sim(O B P))\} .
\end{array}
$$

Then a resolution refutation proof is as follows:

(a) $(M I \vee \sim(O B P))(\sim(O B P) \vee \sim(M I)) \rightarrow \sim(O B P)$,

$$
(\sim(O B P))((O B P) \vee(O C)) \rightarrow O C,(O C)(\sim O C) \rightarrow \square .
$$

(S4) Finally, we claim that $: O B P \rightarrow O W B P$ and $O B P \rightarrow(\sim O W B P)$, are both satisfiable.

Setting $O B P=O W B P=1$ and $M I=O C=0$ the first implication as well as the formula that models the machine shop are satisfiable. The second implication as well as the formula that models the machine shop are also satisfiable assigning: $O B P=1$ and $O W B P=M I=O C=0$. Both conditions are consistent with the Petri net model.

\subsection{The Multirobotic Modeling System Problem}

A flexible manufacturing system is an efficient production line with versatile machines, an automatic transport system and a sophisticated decision making 
system. Flexible manufacturing systems can be formed by subsystems that work concurrently and, therefore are suitable to be modeled by Petri nets.

In this Subsection, the philosophy presented in remark 17 is applied to a multirobotic system, (a particular example of a flexible manufacturing system), in order to validate its Petri net model. The multirobotic system consists of two robot arms which perform pick and place operations accessing a common workspace at times to obtain or transfer parts. In order to avoid collision, it is assumed that only one robot can access the workspace at a time. In addition, it is assumed that the common workspace has a buffer with a limited space for products. the process represents the operation of the two robots serving two different machining tools, with one robot arm transferring products from one machining tool to the buffer, and the other robot arm transferring semiproducts from the buffer to the other machining tool. The Petri net which represents the system is shown in fig. 2 .

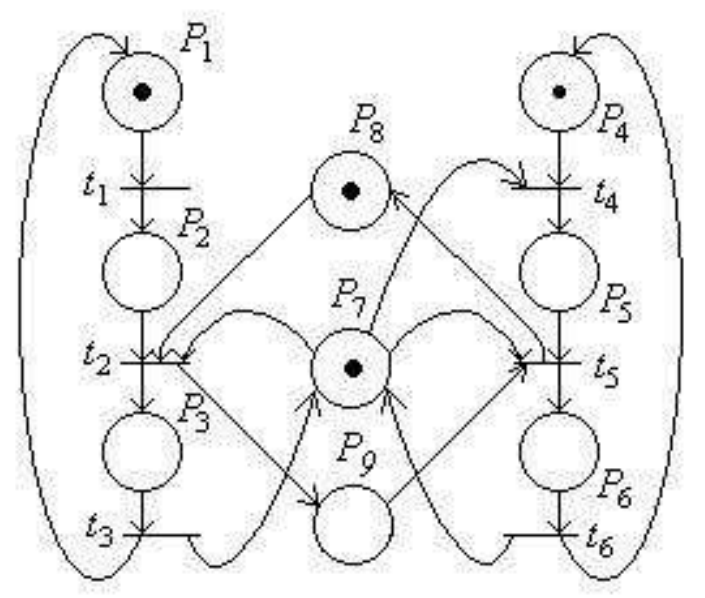

Figure 2. Multirobotic System

Where the interpretation of places and transitions is as follows: 
Place Interpretation

$P_{1}\left(P_{4}\right) \quad$ Robot $\mathrm{R}_{1}\left(\mathrm{R}_{2}\right)$ performs tasks outside the common workspace

$P_{2}\left(P_{5}\right) \quad$ Robot $\mathrm{R}_{1}\left(\mathrm{R}_{2}\right)$ waits for access to the common workspace

$P_{3}\left(P_{6}\right) \quad$ Robot $\mathrm{R}_{1}\left(\mathrm{R}_{2}\right)$ performs in the common workspace

$P_{7} \quad$ mutual exclusion

$P_{8}\left(P_{9}\right) \quad$ number of empty (full) positions in buffer

Transition Interpretation

$t_{1}\left(t_{4}\right) \quad$ Robot $\mathrm{R}_{1}\left(\mathrm{R}_{2}\right)$ requests access to the common workspace

$t_{2}\left(t_{5}\right) \quad$ Robot $\mathrm{R}_{1}\left(\mathrm{R}_{2}\right)$ enters the common workspace

$t_{3}\left(t_{6}\right) \quad$ Robot $\mathrm{R}_{1}\left(\mathrm{R}_{2}\right)$ leaves the common workspace

The multirobotic system behavior as described by the Petri net model is as follows: (1) States $(i=1,2): R_{i} O W P$ Robot $i$ performs tasks outside the common workspace, $R_{i} W A W P$ Robot $i$ waits for access to the common workspace, $R_{i} P W P$ Robot $i$ performs in the working place, $M E$ mutual exclusion, $P B_{i}$ buffers; (2) Rules of Inference: (a) and (b) $(i=1,2) R_{i} O W P \rightarrow R_{i} W A W P$, (c) $R_{1} W A W P \wedge P B_{1} \wedge M E \rightarrow R_{1} P W P \wedge P B_{2}$, (d) $R_{2} W A W P \wedge P B_{2} \wedge M E \rightarrow$ $R_{2} P W P \wedge P B_{1}$, (e) and (f) $(i=1,2) R_{i} P W P \rightarrow M E \wedge R_{i} O W P,(\mathrm{~g})$ and (h) $P B_{1} \leftrightarrow \sim\left(P B_{2}\right)$.

The formula that models the multirobotic system turns out to be:

$$
\begin{aligned}
& {\left[\left(R_{1} O W P\right) \rightarrow\left(R_{1} W A W P\right)\right] \wedge } {\left[\left(R_{2} O W P\right) \rightarrow\left(R_{2} W A W P\right)\right] } \\
& \wedge\left[\left(R_{1} W A W P\right) \wedge\left(P B_{1}\right) \wedge(M E) \rightarrow\left(R_{1} P W P\right) \wedge\left(P B_{2}\right)\right] \wedge\left[\left(R_{2} W A W P\right)\right. \\
&\left.\wedge\left(P B_{2}\right) \wedge(M E) \rightarrow\left(R_{2} P W P\right) \wedge\left(P B_{1}\right)\right] \wedge\left[\left(R_{1} P W P\right)\right. \\
&\left.\rightarrow(M E) \wedge\left(R_{1} O W P\right)\right] \wedge\left[\left(R_{2} P W P\right) \rightarrow(M E) \wedge\left(R_{2} O W P\right)\right] \\
& \wedge\left[P B_{1} \rightarrow \sim\left(P B_{2}\right)\right] \wedge\left[\sim\left(P B_{2}\right) \rightarrow P B_{1}\right] .
\end{aligned}
$$

We are interested in the following validations:

(S1) Claim: $R_{1} O W P \wedge P B_{1} \wedge M E \rightarrow R_{1} P W P$.

The set of clauses is given by:

$$
\begin{gathered}
S= \\
\left\{\left(\sim\left(R_{1} O W P\right) \vee\left(R_{1} W A W P\right)\right),\left(\sim\left(R_{2} O W P\right) \vee\left(R_{2} W A W P\right)\right),\left(\sim\left(R_{1} W A W P\right)\right.\right. \\
\left.\vee \sim\left(P B_{1}\right) \vee \sim(M E) \vee\left(R_{1} P W P\right)\right),\left(\sim\left(R_{1} W A W P\right) \vee \sim\left(P B_{1}\right)\right. \\
\left.\vee \sim(M E) \vee\left(P B_{2}\right)\right),\left(\sim\left(R_{2} W A W P\right) \vee \sim\left(P B_{2}\right) \vee \sim(M E)\right. \\
\left.\vee\left(R_{2} P W P\right)\right),\left(\sim\left(R_{2} W A W P\right) \vee \sim\left(P B_{2}\right) \vee \sim(M E) \vee\left(P B_{1}\right)\right), \\
\left(\sim\left(R_{1} P W P\right) \vee(M E)\right),\left(\sim\left(R_{1} P W P\right) \vee\left(R_{1} O W P\right)\right),\left(\sim\left(R_{2} P W P\right) \vee(M E)\right), \\
\left(\sim\left(R_{2} P W P\right) \vee\left(R_{2} O W P\right)\right),\left(P B_{1} \vee P B_{2}\right),\left(\sim\left(P B_{1}\right) \vee \sim\left(P B_{2}\right)\right),
\end{gathered}
$$




$$
\left.\left(R_{1} O W P\right),\left(P B_{1}\right),(M E),\left(\sim\left(R_{1} P W P\right)\right)\right\} .
$$

Then a resolution refutation proof is as follows:

(a) $\left(R_{1} O W P\right)\left(\sim\left(R_{1} O W P\right) \vee\left(R_{1} W A W P\right)\right) \rightarrow\left(\left(R_{1} W A W P\right)\right)$;

(b) $\left(\sim\left(R_{1} W A W P\right) \vee \sim\left(P B_{1}\right) \vee \sim(M E)\right.$

$$
\left.\vee\left(R_{1} P W P\right)\right)\left(\left(\left(R_{1} W A W P\right)\right)\left(P B_{1}\right)(M E) \rightarrow\left(R_{1} P W P\right) ;\right.
$$

(c) $\left(R_{1} P W P\right)\left(\sim\left(R_{1} P W P\right)\right) \rightarrow \square$.

(S2) Claim: $R_{1} W A W P \wedge P B_{1} \wedge M E \rightarrow P B_{2}$.

The set of clauses is given by:

$$
\begin{gathered}
S=\left\{\left(\sim\left(R_{1} O W P\right) \vee\left(R_{1} W A W P\right)\right),\left(\sim\left(R_{2} O W P\right) \vee\left(R_{2} W A W P\right)\right),\right. \\
\left(\sim\left(R_{1} W A W P\right) \vee \sim\left(P B_{1}\right) \vee \sim(M E) \vee\left(R_{1} P W P\right)\right),\left(\sim\left(R_{1} W A W P\right)\right. \\
\left.\quad \vee \sim\left(P B_{1}\right) \vee \sim(M E) \vee\left(P B_{2}\right)\right),\left(\sim\left(R_{2} W A W P\right) \vee \sim\left(P B_{2}\right)\right. \\
\left.\vee \sim(M E) \vee\left(R_{2} P W P\right)\right),\left(\sim\left(R_{2} W A W P\right) \vee \sim\left(P B_{2}\right) \vee \sim(M E)\right. \\
\left.\vee\left(P B_{1}\right)\right),\left(\sim\left(R_{1} P W P\right) \vee(M E)\right),\left(\sim\left(R_{1} P W P\right) \vee\left(R_{1} O W P\right)\right), \\
\left(\sim\left(R_{2} P W P\right) \vee(M E)\right),\left(\sim\left(R_{2} P W P\right) \vee\left(R_{2} O W P\right)\right),\left(P B_{1} \vee P B_{2}\right), \\
\left.\left(\sim\left(P B_{1}\right) \vee \sim\left(P B_{2}\right)\right),\left(R_{1} W A W P\right),\left(P B_{1}\right),(M E),\left(\sim\left(P B_{2}\right)\right)\right\} .
\end{gathered}
$$

Then a resolution refutation proof is as follows:

$$
\begin{aligned}
\left(\sim\left(R_{1} W A W P\right) \vee \sim\left(P B_{1}\right) \vee \sim(M E) \vee\right. & \left.\left(P B_{2}\right)\right)\left(\left(R_{1} W A W P\right)\left(P B_{1}\right)(M E)\right. \\
& \left.\left.\rightarrow\left(P B_{2}\right)\right),\left(P B_{2}\right)\right)\left(\sim\left(P B_{2}\right)\right) \rightarrow \square .
\end{aligned}
$$

(S3) Next, we validate the mutual exclusive property of the Petri net model.

Claim. $R_{1} W A W P \wedge R_{2} W A W P \wedge M E \rightarrow\left(R_{1} P W P\right.$

$$
\left.\vee R_{2} P W P\right) \wedge \sim\left(R_{1} P W P \wedge R_{2} P W P\right) .
$$

The set of clauses is given by:

$$
\begin{aligned}
S=\{( & \left.\left(R_{1} O W P\right) \vee\left(R_{1} W A W P\right)\right),\left(\sim\left(R_{2} O W P\right) \vee\left(R_{2} W A W P\right)\right), \\
\left(\sim\left(R_{1} W A W P\right) \vee \sim\left(P B_{1}\right) \vee \sim(M E) \vee\left(R_{1} P W P\right)\right),\left(\sim\left(R_{1} W A W P\right)\right. & \left.\vee \sim\left(P B_{1}\right) \vee \sim(M E) \vee\left(P B_{2}\right)\right),\left(\sim\left(R_{2} W A W P\right) \vee \sim\left(P B_{2}\right)\right. \\
& \left.\vee \sim(M E) \vee\left(R_{2} P W P\right)\right),\left(\sim\left(R_{2} W A W P\right) \vee \sim\left(P B_{2}\right) \vee \sim(M E)\right. \\
& \left.\vee\left(P B_{1}\right)\right),\left(\sim\left(R_{1} P W P\right) \vee(M E)\right),\left(\sim\left(R_{1} P W P\right) \vee\left(R_{1} O W P\right)\right), \\
& \left(\sim\left(R_{2} P W P\right) \vee(M E)\right),\left(\sim\left(R_{2} P W P\right) \vee\left(R_{2} O W P\right)\right),\left(P B_{1}\right.
\end{aligned}
$$




$$
\begin{array}{r}
\left.\vee P B_{2}\right),\left(\sim\left(P B_{1}\right) \vee \sim\left(P B_{2}\right)\right),\left(R_{1} W A W P\right),\left(R_{2} W A W P\right),(M E), \\
\left.\left(\sim\left(R_{1} P W P\right)\right),\left(\sim\left(R_{2} P W P\right)\right)\right\} .
\end{array}
$$

Then a resolution refutation proof is as follows:

(a) $\left(\sim\left(R_{1} W A W P\right) \vee \sim\left(P B_{1}\right) \vee \sim(M E) \vee\left(R_{1} P W P\right)\right)\left(R_{1} W A W P\right)(M E)(\sim$ $\left.\left(R_{1} P W P\right)\right) \rightarrow\left(\sim\left(P B_{1}\right)\right)$

(b) $\left(\sim\left(R_{2} W A W P\right) \vee \sim\left(P B_{2}\right) \vee \sim(M E) \vee\left(R_{2} P W P\right)\right)\left(R_{2} W A W P\right)(M E)(\sim$ $\left.\left(R_{2} P W P\right)\right) \rightarrow\left(\sim\left(P B_{2}\right)\right)$

(c) $\left.\left(\sim\left(P B_{1}\right)\right)\left(P B_{1}\right) \vee\left(P B_{2}\right)\right) \rightarrow\left(P B_{2}\right)$;

(d) Then, from (b) and (c) we get $\square$.

(S4) Claim: $R_{1} P W P \wedge P B_{2} \wedge R_{2} W A W P \rightarrow R_{2} P W P \wedge R_{1} O W P \wedge M E \wedge$ $P B_{1}$

The set of clauses is given by:

$$
\begin{gathered}
S=\left\{\left(\sim\left(R_{1} O W P\right) \vee\left(R_{1} W A W P\right)\right),\left(\sim\left(R_{2} O W P\right) \vee\left(R_{2} W A W P\right)\right),\right. \\
\left(\sim\left(R_{1} W A W P\right) \vee \sim\left(P B_{1}\right) \vee \sim(M E) \vee\left(R_{1} P W P\right)\right), \\
\left(\sim\left(R_{1} W A W P\right) \vee \sim\left(P B_{1}\right) \vee \sim(M E) \vee\left(P B_{2}\right)\right), \\
\left(\sim\left(R_{2} W A W P\right) \vee \sim\left(P B_{2}\right) \vee \sim(M E) \vee\left(R_{2} P W P\right)\right), \\
\left(\sim\left(R_{2} W A W P\right) \vee \sim\left(P B_{2}\right) \vee \sim(M E) \vee\left(P B_{1}\right)\right), \\
\left(\sim\left(R_{1} P W P\right) \vee(M E)\right),\left(\sim\left(R_{1} P W P\right) \vee\left(R_{1} O W P\right)\right),\left(\sim\left(R_{2} P W P\right) \vee(M E)\right), \\
\left(\sim\left(R_{2} P W P\right) \vee\left(R_{2} O W P\right)\right),\left(P B_{1} \vee P B_{2}\right),\left(\sim\left(P B_{1}\right) \vee \sim\left(P B_{2}\right)\right), \\
\left(R_{1} P W P\right),\left(P B_{2}\right),\left(R_{2} W A W P\right),\left(\sim\left(R_{2} P W P\right) \vee \sim\left(R_{1} O W P\right)\right. \\
\left.\left.\vee \sim(M E) \vee \sim\left(P B_{1}\right)\right)\right\} .
\end{gathered}
$$

Then a resolution refutation proof is as follows:

(a) $\left(R_{1} P W P\right)\left(\sim\left(R_{1} P W P\right) \vee(M E)\right) \rightarrow(M E)$;

(b) $\left(R_{1} P W P\right)\left(\sim\left(R_{1} P W P\right) \vee\left(R_{1} O W P\right)\right) \rightarrow\left(R_{1} O W P\right)$;

(c) $\left(\sim\left(R_{2} W A W P\right) \vee \sim\left(P B_{2}\right) \vee \sim(M E) \vee\left(P B_{1}\right)\right)\left(R_{2} W A W P\right)\left(P B_{2}\right)(M E)$ $\rightarrow\left(P B_{1}\right)$

(d) $\left(\sim\left(R_{2} P W P\right) \vee \sim\left(R_{1} O W P\right) \vee \sim(M E)\right.$

$$
\left.\vee \sim\left(P B_{1}\right)\right)(M E)\left(R_{1} O W P\right)\left(P B_{1}\right) \rightarrow\left(\sim\left(R_{2} P W P\right)\right) ;
$$

(e) $\left(\sim\left(R_{2} W A W P\right) \vee \sim\left(P B_{2}\right) \vee \sim(M E)\right.$

$$
\left.\vee\left(R_{2} P W P\right)\right)\left(R_{2} W A W P\right)\left(P B_{2}\right)\left(\sim\left(R_{2} P W P\right)\right) \rightarrow(\sim(M E)) ;
$$

Then, from (a) and (e) we get $\square$. 


\section{Conclusions}

The main contribution of the paper consists in proposing a formal reasoning deductive methodology based on the propositional calculus logic theory for validation of Petri net models of discrete event systems without having to construct its state space.

\section{References}

[1] J.A. Robinson, A machine-oriented logic based on the resolution principle, Journal of the $A C M, \mathbf{1 2}$, No. 1 (1965), 23-41.

[2] M. Davis, R. Sigal and E. Weyuker, Computability, Complexity, and Languages, Fundamentals of Theoretical Computer Science, Academic Press, 1983.

[3] J. H. Gallier, Logic For Computer Science Automatic Theorem Proving, Dover, 2015. 
